\title{
miR-577 suppresses cell proliferation and epithelial-mesenchymal transition by regulating the WNT2B mediated Wnt/B-catenin pathway in non-small cell lung cancer
}

\author{
BIN WANG, LIWEI SUN, JINDUO LI and RONG JIANG \\ Department of Interventional Radiology, Tianjin Huanhu Hospital, Tianjin 300350, P.R. China
}

Received October 10, 2017; Accepted March 7, 2018

DOI: $10.3892 / \mathrm{mmr} .2018 .9279$

\begin{abstract}
MicroRNAs (miRNAs/miRs) serve important roles in regulating malignant phenotype in numerous cancers, such as non-small cell lung cancer (NSCLC); however, the role and function of miR-577 in NSCLC remains unknown. In the present study, miR-577 expression levels were observed to be downregulated in NSCLC via reverse transcriptionquantitative polymerase chain reaction (RT-qPCR) assay, and inhibited cell proliferation, cell migration and invasion and epithelial-mesenchymal transition progress in NSCLC cells. The predicted target genes of miR-577 were determined by enhanced green fluorescent protein reporter assay, RT-qPCR and western blot analyses. miR-577 was demonstrated to suppress the expression of WNT2B by targeting the 3'-untranslated region of WNT2B mRNA in H522 and A549 cells. WNT2B was upregulated in NSCLC cells as observed via RT-qPCR analysis, and the malignant phenotype of H522 and A549 cells were promoted by WNT2B overexpression. In addition, miR-577 inactivated the Wnt/ $\beta$-catenin pathway by targeting WNT2B in NSCLC cells. Collectively, miR-577 may function as a suppressor gene by directly downregulatingWNT2B mRNA and protein expression levels in H522 and A549 cells, and may serve important roles in the malignancy of NSCLC.
\end{abstract}

\section{Introduction}

Lung cancer is one of the most common malignancies, which is the leading cause of cancer-associated mortality worldwide (1). Non-small cell lung cancer (NSCLC), including squamous cell carcinoma, adenocarcinoma and large cell carcinoma are the most common types of lung cancer (2). Notably, NSCLC

Correspondence to: Dr Rong Jiang or Dr Bin Wang, Department of Interventional Radiology, Tianjin Huanhu Hospital, 6 Jizhao Road, Jinnan, Tianjin 300350, P.R. China

E-mail: 15900333030@163.com

E-mail: ourdongzi@163.com

Key words: microRNA-577, Wnt family member 2B, non-small cell lung cancer, Wnt//-catenin, epithelial-mesenchymal transition constitutes $\sim 85 \%$ of general lung cancers $(3,4)$. Notable improvements have been made in the treatment of NSCLC, including surgical resection, chemotherapy, radiation therapy or a combination of targeted therapy over the past several decades, the prognosis of patients with NSCLC remains unfavorable; the total survival rate is $\sim 15 \%(5,6)$. Furthermore, the majority of patients are diagnosed at the later period of lung cancer, which greatly decreases the survival outcome (7). Thus, investigating the mechanism of development and progression of NSCLC and identifying novel therapeutic strategies for NSCLC is urgently required.

MicroRNAs (miRNAs/miRs) function as small noncoding RNAs of 18-25 nucleotides that negatively regulate gene expression by affecting translational repression or mRNA cleavage at the post-transcriptional level via complementarities with the 3'untranslated region (UTR) of their target genes $(8,9)$. Dysregulation of miRNA expression is often associated with a variety of human malignancies and increasing evidence indicates that miRNAs may function as either oncogenes or tumor suppressors $(9,10)$. At present, numerous miRNAs have been reported to be upregulated or downregulated in NSCLC (11-13). For example, Sun et al (14) reported that miR-503-3p inhibits lung cancer cell viability and induces cell apoptosis by regulating p21 and cyclin dependent kinase 4 expression in lung cancer cells. In addition, Li et al (15) reported that ectopic expression of miR-146b-5p suppresses cell proliferation, clonogenicity, migration and invasion, and also induces G1 arrest in vitro, but does not induce cell apoptosis by regulating matrix metalloproteinase 16 and tumor necrosis factor receptor associated factor 6 expression in NSCLC. Furthermore, Huang et al (16) reported that miR219-5p exerts the tumor-suppressive function by inhibiting the activation of the protein kinase B (AKT) and extracellular signal-regulated kinase (ERK) pathways in NSCLC cells; however, the role and mechanism of regulation of miR-577 in NSCLC remain unclear.

In the present study, miR-577 was demonstrated to be downregulated in NSCLC tissues and cell lines; low miR-577 expression levels were associated with larger tumor size, advanced tumor, node, metastasis (TNM) stage and lymph node metastasis of patients with NSCLC. Functional analysis revealed that miR-577 overexpression promoted cell proliferation. In addition, Transwell analysis revealed that the inhibitory effect of miR-577 overexpression on cell migration and invasion 
functions by inhibiting the epithelial-mesenchymal transition (EMT) process in NSCLC cells. Furthermore, Wnt family member 2B (WNT2B) may be a target of miR-577 and serves the oncogenic role in NSCLC progression by activating the Wnt/ $\beta$-catenin signaling pathway. Collectively, the findings of the present study suggested that miR-577 may inhibit NSCLC progression via the direct targeting of WNT2B; the Wnt/ $\beta$ catenin signaling pathway may be involved in the regulatory mechanism.

\section{Materials and methods}

Tissue samples. A total of 25 NSCLC tissues and the adjacent normal lung tissues were obtained from patients $(n=25$; 13 male and 12 female; aged 39-78 years) admitted to Tianjin Huanhu Hospital (Tianjin, China) between March 2013 and March 2016. All of the samples were obtained with the patients' informed consent. The entire investigation conformed to the principles outlined in The Declaration of Helsinki. The present study was approved by the ethical review committees of Tianjin Huanhu Hospital.

Cell cultures. Human NSCLC cell lines, including H650, A549, H522, H1299 and H1155 were purchased from the Cell Bank of Type Culture Collection of the Chinese Academy of Sciences (Shanghai, China), and human normal bronchial epithelial cells (HBECs) were purchased from Shanghai Maisha Biotechnology (http://maishabio.biogo.net/; Shanghai, China). The cells were routinely grown in Dulbecco's modified Eagle's medium (DMEM; Invitrogen; Thermo Fisher Scientific, Inc., Waltham, MA, USA) supplemented with $10 \%$ fetal bovine serum (FBS; Gibco; Thermo Fisher Scientific, Inc.), $1 \%$ penicillin/streptomycin mix (Sigma-Aldrich; Merck KGaA, Darmstadt, Germany), 2 mM glutamine (Gibco; Thermo Fisher Scientific, Inc.), 5 mM glucose (Sigma Aldrich; Merck KGaA) and $1 \mathrm{mM}$ sodium pyruvate (Sigma Aldrich; Merck $\mathrm{KGaA}$ ) at $37^{\circ} \mathrm{C}$ in a humidified atmosphere containing $5 \% \mathrm{CO}_{2}$.

Reverse transcription-quantitative polymerase chain reaction $(R T-q P C R)$ assay. Total RNA was extracted from cultured cells and NSCLC tissues using TRIzol ${ }^{\circledR}$ reagent (Invitrogen; Thermo Fisher Scientific, Inc.) according to the manufacturer's protocol. miRNAs from cancer specimens or cells were extracted using an RNeasy kit or miRNeasy mini kit (Qiagen GmbH, Hilden, Germany), respectively, according to the manufacturer's protocols. miRNAs and mRNAs were reverse transcribed using a miScript reverse transcription kit (Qiagen $\mathrm{GmbH}$ ) following the manufacturer's protocols. qPCR was performed using a miRNA-specific TaqMan MiRNA Assay kit (Applied Biosystems; Thermo Fisher Scientific, Inc.) according to the manufacturer's protocols using a Applied Biosystems 7500 Fast Real-Time PCR system (Thermo Fisher Scientific, Inc.). The qPCR conditions were as follows: $94^{\circ} \mathrm{C}$ pre-denaturation for $5 \mathrm{~min}$, followed by 33 cycles of denaturation at $94^{\circ} \mathrm{C}$ for $30 \mathrm{sec}$, annealing and synthesis at $58^{\circ} \mathrm{C}$ for $30 \mathrm{sec}$. Relative gene expression data was analyzed using the $2^{-\Delta \Delta \mathrm{Cq}}$ method (17). The primers used for RT-qPCR were as follows: miR-577-RT 5'-GTCGTATCCAGTGCAGGGTCC GAGGTGCACTGGATACGACCAGGTA-3'; oligod T 5'-TTT
TTTTTTTTTTTTTTT-3'; U6-RT 5'-GTCGTATCCAGTGCA GGGTCCGAGGTGCACTGGATACGACAAAATATGG-3'; miR-577-qPCR, forward 5'-TGCGGTAGATAAAATATT GG-3', reverse 5'-GTGCAGGGTCCGAGGT-3'; U6-qPCR, forward 5'-GCTTCGGCAGCACATATACTAAAAT-3', reverse 5'-CGCTTCACGAATTTGCGTGTCAT-3'; WNT2BqPCR, forward 5'-GCTGGACCAAACCTGAAC-3', reverse 5'-CAAGAAGTATCGGGAAGC-3'; and $\beta$-actin-qPCR, forward 5'-CCGTCTTCCCCTCCATCGTGGG-3', reverse 5'-CGCAGCTCATTGTAGAAGGTGTGG-3'.

Plasmid construction. WNT2B was overexpressed using PCR-amplified cDNA of H522 cells, which was cloned between the KpnI and XbaI restriction sites into the pcDNA3 vector (Beyotime Institute of Biotechnology, Shanghai, China). Overexpression was confirmed by RT-qPCR and western blot analysis. The pcDNA3 vector alone was used as the control group. In order to overexpress miR-577, the primary miR-577 was amplified from genomic DNA of H522 cells and cloned into the pcDNA3 vector between the BamHI and EcoRI restriction sites. To inhibit the function of miR-577, the 2-O-methyl-modifed antisense oligonucleotide of miR-577 (ASO-miR-577) and the scramble control oligonucleotides (ASO-NC) from the Shanghai GenePharma, Co., Ltd. (Shanghai, China) were used. The pSilencer/shR-WNT2B (shR-WNT2B) plasmid expressing siRNA targeting WNT2B was constructed by annealing double-stranded hairpin cDNA and inserting it into the pSilencer 2.1-U6 neo vector (Ambion; Thermo Fisher Scientific, Inc.) at the BamHI and EcoRI sites. The primers and sequences used are listed as follows: Pri-miR-577-sense (S) 5'-CGGGGTACCTGGTAG GTGCCCTGTTGA-3', pri-miR-577-antisense (AS) 5'-CCG GAATTCTGGAAAGTA ACCACGAGA-3'; ASO-miR-577 5'-CAGGUACCAAUAUUUUAUCUA-3'; ASO-NC 5'-UCA CAACCUCCUAGAAAGAGUAGA-3'; WNT2B-S 5'-CGG GGTACCGCCACCATGTTGGATGGCCTTGGAGTG-3'; and WNT2B-AS 5'-TGCTCTAGATCAGGTTTGGTCCAG CCACTCTGCC-3'; shR-WNT2B-S 5'-GATCCCGGACTG ATCTTGTCTACTTTCTCGAGAAAGTAGACAAGATCA GTCCGTTTTTGA-3'; shR-WNT2B-AS 5'-AGCTTCCGG ACTGATCTTGTCTACTTTCTCGAGAAAGTAGACAAG ATCAGTCCGG-3'. For transfection, H522 and A549 cells were seeded in 12-well plates at a density of $1 \times 10^{7}$ cells $/ \mathrm{ml}$ for $24 \mathrm{~h}$, and were subsequently transfected with $2 \mu \mathrm{g}$ plasmids using Lipofectamine ${ }^{\circledR} 2000$ (DNA:Lipofectamine ${ }^{\circledR} 2000=1: 2$; Invitrogen; Thermo Fisher Scientific, Inc.) according to the manufacturer's protocols and incubated for $4 \mathrm{~h}$ at $37^{\circ} \mathrm{C}$. Subsequently, the cells were cultured in DMEM supplemented with $10 \% \mathrm{FBS}$ for $48 \mathrm{~h}$ at $37^{\circ} \mathrm{C}$ prior to further analysis.

Prediction of miRNA targets. The hypothetical target of miR-577 was predicted using miRDB (http://www.mirdb. org/) (18), miRNA.org and TargetScan human7.1 (http://www. targetscan.org/vert_71/), which revealed that the 3'UTR of WNT2B may be complementarily paired with the seed sequences of miR-577.

Enhanced green fluorescent protein (EGFP) reporter assay. The WNT2B 3'UTR was cloned into a pcDNA3/EGFP vector (Shanghai GeneChem Co.,Ltd., Shanghai,China) and mutations 
were introduced at potential miR-577 binding sites. To determine that the 3'UTR of WNT2B mRNA is directly targeted by miR-577, 2x10 ${ }^{6}$ H522 and A549 cells were cotransfected with $0.5 \mu \mathrm{g}$ pri-miR-577 or $20 \mathrm{nM}$ ASO-miR-577 and $0.5 \mu \mathrm{g}$ 3'UTR of WNT2B or the mutant 3'UTR of WNT2Bin in 48-well plates using Lipofectamine ${ }^{\circledR} 2000$ (DNA:Lipofectamine ${ }^{\circledR}$ $2000=1: 2$; Invitrogen; Thermo Fisher Scientific, Inc.). The binding site of miR-577 in the WNT2B 3'UTR was mutated as follows: 5' UAACAUUAUUAACAUUUAGAA3'. After 48 h, the EGFP activity was measured using a spectrophotometer set at $528 \mathrm{~nm}$. Red fluorescent protein-expressing plasmid was integrated as a transfection efficiency control.

CCK-8 assay. H522 and A549 cells transfected as aforementioned were seeded in 96-well plates at a density of $5 \times 10^{3}$ cells/well. A Cell Counting kit-8 (CCK-8; Dojindo Molecular Technologies, Inc., Kumamoto, Japan) was used to detect the viability of H522 and A549 cells according to the manufacturer's instructions. The absorbance at $450 \mathrm{~nm}$ was measured.

Colony formation assay. For the colony formation ability assay, H522 and A549 cells were counted at $24 \mathrm{~h}$ post-transfection and seeded into 24 -well plates at 500 cell/well. Culture medium was replaced every 3 days. After $\sim 2$ weeks, cells were washed with 1 XPBS. Subsequently, colonies were fixed with $4 \%$ paraformaldehyde at room temperature for 30 min and stained with $1 \%$ crystal violet at room temperature for $20 \mathrm{~min}$. The number of colonies was counted under an inverted microscope (Leica Microsystems GmbH, Wetzlar, Germany).

Transwell migration and invasion assay. H522 and A549 cells transfected with the indicated plasmids were collected and suspended in serum-free medium. Subsequently, 6x105 cells were added to the upper chamber covered with $12.5 \mathrm{mg}$ Matrigel (BD Biosciences) in $50 \mathrm{ml}$ PBS, the lower chamber was filled with medium containing $20 \%$ FBS. Following incubation at $37^{\circ} \mathrm{C}$ for $48 \mathrm{~h}$, cells below the membrane were fixed and stained with $0.5 \%$ crystal violet at room temperature for 30 min, washed with $1 \mathrm{X}$ PBS, air-dried and observed under an inverted microscope (Olympus Corporation, Tokyo, Japan). Cell invasion was assessed using a Transwell system (Corning Incorporated, Tewksbury, MA, USA). The number of migrated and invaded cells was counted under a microscope.

Western blot analysis. A total of $6 \times 10^{8}$ A549 cells were lysed using the Protein Extraction kit according to the manufacturer's protocols (http://www.wanleibio.cn/; Wanlei Biotechnology, Beijing, China); $30 \mu \mathrm{g}$ total proteins were separated by $10 \%$ SDS-PAGE and transferred onto nitrocellulose membranes (EMD Millipore, Billerica, MA, USA). The membranes were blocked with $5 \%$ non-fat milk in Tris-buffered saline containing Tween-20 for $\sim 2$ hat room temperature, prior to incubation with the primary antibodies. The membranes were probed with anti-E-cadherin (1:1,500; cat. no. ab1416; Abcam, Cambridge, MA, USA), anti-intercellular adhesion molecule 1 (ICAM1; 1:3,000; cat. no. ab223659; Abcam), antiVimentin (1:2,000; cat. no. ab188499; Abcam), anti-WNT2B (1:2,000; cat. no. ab50575; Abcam), anti- $\beta$-catenin $(1: 5,000$; cat. no. ab16051; Abcam), anti-cyclin D1 (1:5,000; cat. no. ab15196; Abcam), anti-c-Myc (1:5,000; cat. no. ab39688; Abcam), p-GSK3 $\beta$ (1:1,000; cat. no. ab131097; Abcam), totalGSK3 $\beta$ (1:3,000; cat. no. ab2602; Abcam) and anti-GAPDH (1:5,000; cat. no. ab9485; Abcam) antibodies overnight at $4^{\circ} \mathrm{C}$. Subsequently, membranes were incubated with a horseradish peroxidase-conjugated secondary antibody (1:5,000; cat. no. 8889; Cell Signaling Technology, Inc., Danvers, MA, USA) for $1 \mathrm{~h}$ at $37^{\circ} \mathrm{C}$. An enhanced chemiluminescence system (Thermo Fisher Scientific, Inc.) was used to detect the immunoreactive bands. The relative protein expression levels were normalized to that of GAPDH. The protein expression levels were measured using Image Pro Plus software v.6.0 (Media Cybernetics, Inc., Rockville, MD, USA).

TOP/FOP flash reporter assays. To determine the transcriptional activity of the Wnt pathway, 6×10 ${ }^{6}$ A549 and H522 cells treated as indicated were co-transfected with either the Wnt signaling reporter TOP Flash or the negative control FOP Flash (EMD Millipore) according to the manufacturer's protocols. A549 and H522 cells were transiently transfected with either $2 \mu \mathrm{g}$ pTOP flash or pFOP flash plasmids and $0.5 \mu \mathrm{g}$ pSV40Renilla plasmid as an internal control (Promega Corporation, Madison, WI, USA) using Lipofectamine ${ }^{\circledR} 2000$ (Invitrogen; Thermo Fisher Scientific, Inc.) at room temperature for $48 \mathrm{~h}$. The dual luciferase reporter assay system (Dual-Luciferase ${ }^{\circledR}$ Reporter Assay system; cat. no. E1910; Promega Corporation) was used to assay the firefly and Renilla luciferase activity ratio.

Immunofluorescence staining. A549 cells transfected with specific plasmids were seeded in 24-well plates at the indicated time points before immunofluorescence staining. The cells were washed in PBS and fixed with $4 \%$ paraformaldehyde for $30 \mathrm{~min}$ at room temperature. After cells were washed with PBS, the cells were permeabilized using $0.25 \%$ Triton-X-100 for $5 \mathrm{~min}$ at room temperature and blocked in $10 \%$ donkey serum (BeyotimeInstitute of Biotechnology; Nanjing, China) for $30 \mathrm{~min}$. The cells were subsequently incubated with primary antibodies against $\beta$-catenin (1:100; cat. no. ab16051; Abcam) overnight at $4^{\circ} \mathrm{C}$. The following day, cells were washed in PBS and then incubated at room temperature for $1 \mathrm{~h}$ with a fluorescent-labeled secondary antibody (1:200; cat. no. A0562; BeyotimeInstitute of Biotechnology), followed by incubation with DAPI (1:1,000; cat. no. C1002; BeyotimeInstitute of Biotechnology). Images were captured under a confocal microscope.

Statistical analysis. All analyses were performed using SPSS v. 19.0 for Windows (IBM Corp., Armonk, NY, USA) and GraphPad Prism v. 5.0 for Windows (GraphPad Software Inc., La Jolla, CA, USA). For comparisons of two treatment groups, a Student's t-test was used. For comparisons of three or more groups, one-way analysis of variance was followed by the Bonferroni post hoc test for comparison of two selected treatment groups; the Dunnett's post hoc test was used for comparisons of the other treatment groups with the corresponding controls. The Pearson's correlation analysis was used to determine the r-value. Associations between miR-577 expression and clinicopathological characteristics were 
assessed using chi-squared test. Data from at least three independent experiments are presented as the means \pm standard deviation, or medians with ranges. $\mathrm{P}<0.05$ was considered to indicate a statistically significant difference.

\section{Results}

miR-577 is downregulated in NSCLC tissues and cell lines. The relative expression levels of miR-577 in NSCLC tissues and cells were measured via RT-qPCR. The results revealed that miR-577 expression levels were significantly lower in NSCLC tissues than that in the adjacent normal tissues (Fig. 1A). In addition, the miR-577 expression levels in NSCLC patients were inversely associated with the tumor size, TNM stage and lymph node metastasis, but not the histological grade, age and gender (Table I). Furthermore, the expression levels of miR-577 were also detected in various human NSCLC cells lines including H650, A549, H522, H1299 and H1155 cells. As presented in Fig. 1B, miR-577 expression levels were lower in NSCLC cell lines compared with HBECs (Fig. 1B). These results suggested that miR-577 may have a suppressive role in NSCLC progression.

miR-577 inhibits cell proliferation, cell migration, cell invasion and EMT processes in NSCLC cells. To further confirm whether miR-577 may affect NSCLC tumorigenesis, pcDNA3, pri-miR-577, ASO-NC or ASO-miR-577 was transfected into H522 and A549 cells. The results of RT-qPCR analysis demonstrated that the expression plasmids were effective (Fig. 1C). CCK-8 and colony formation assays revealed that the overexpression of miR-577 significantly inhibited cell proliferation and knockdown of miR-577 significantly promoted cell proliferation of H522 and A549 cells (Fig. 1D and E). Transwell migration and invasion assays revealed that ectopic expression of miR-577 significantly reduced the cell migration and invasion ability of H522 and A549 cells; knockdown of miR-577 increased H522 and A549 cells migration and invasion (Fig. 1F and G). These suggest that miR-577 may have a suppressive effect on NSCLC metastasis. In order to investigate the regulation of EMT by miR-577, the expression levels of the EMT markers, E-cadherin, Vimentin and ICAM-1 were analyzed by western blotting in A549 cells and the results indicated that the expression levels of Vimentin and ICAM-1 were significantly decreased by miR-577 overexpression but increased by miR-577 knockdown compared to the control groups; in addition, the expression levels of E-cadherin were significantly increased by $\mathrm{miR}-577$ overexpression, but attenuated by miR-577 knockdown (Fig. 1H).

WNT2B is a direct target of $m i R-577$. To investigate the mechanism of miR-577 affecting the biological behavior in NSCLC cells, the target genes of miR-577 were predicted using miRDB, miRNA.org and TargetScan human7.1; WNT2B was predicted to be a target of miR-577 (Fig. 2A). To verify that miR-577 can directly target WNT2B mRNA, EGFP reporter plasmids containing the 3'UTR or the 3'UTR-mut of WNT2B were constructed. In H522 and A549 cells, the relative EGFP level was significantly reduced in the pri-miR-577 and wild type 3'UTR of WNT2B co-transfected group. Additionally, co-transfection with ASO-miR-577 and wild type 3'UTR of
WNT2B significantly increased the relative EGFP activity (Fig. 2B); however, no significant differences in EGFP activity were observed when H522 and A549 cells were co-transfected with pri-miR-577 or ASO-miR-577 and mutational EGFP reporter plasmids (Fig. 2C). To further confirm the regulation of WNT2B by miR-577, RT-qPCR and western blot assays were performed. The results revealed that overexpression of miR-577 significantly decreased the mRNA and protein levels of WNT2B; miR-577 knockdown significantly increased the expression of WNT2B at the mRNA and protein levels, suggesting that miR-577 downregulated WNT2B expression at the mRNA and protein levels in H522 and A549 cells (Fig. 2D and E). In addition, the present study reported that the mRNA levels of WNT2B in tumor tissues were negatively correlated with the miR-577 levels using Pearson's correlation analysis ( $\mathrm{r}=-0.8743, \mathrm{R}^{2}=0.7644$; Fig. $\left.2 \mathrm{~F}\right)$. These data indicated that WNT2B may be negatively regulated by miR-577 in NSCLC cells, which is the novel target of miR-577.

Upregulated WNT2B promotes the malignancy of NSCLC cells. To determine the role of WNT2B in the aggressiveness of NSCLC, the mRNA expression levels of WNT2B in NSCLC cell lines were analyzed by RT-qPCR. The results demonstrated that WNT2B expression levels were significantly upregulated in NSCLC cells compared with in HBECs (Fig. 3A). In addition, the protein expression levels of WNT2B were detected by western blot assays, which also revealed that WNT2B was increased in NSCLC cells compared with in HBECs cells (Fig. 3B). As presented in Fig. 3C and D, the overexpression or knockdown of WNT2B were effective in H522 and A549 cells. At 24, 48 and $72 \mathrm{~h}$ post-transfection, cell viabilities of H522 and A549 cells were significantly increased by WNT2B overexpression and decreased by WNT2B knockdown (Fig. 3E). In addition, the relative colony formation rate was significantly increased by pWNT2B-transfected and decreased by shR-WNT2B-transfected compared with in the control groups (Fig. 3F). To identify whether WNT2B also influences the migration and invasion of NSCLC cells, Transwell migration and invasion assays were performed in H522 and A549 cells. As expected, overexpression of WNT2B significantly promoted cell migration and invasion ability (Fig. 3G and H). Finally, whether WNT2B may regulate the protein expression levels of the EMT process was investigated, including E-cadherin, ICAM-1 and Vimentin. Western blot analysis demonstrated that the overexpression of WNT2B significantly decreased E-cadherin expression, but increased ICAM-1 and Vimentin expression levels in A549 cells (Fig. 3I). Collectively, these results indicated that upregulation of WNT2B promotes NSCLC malignant behavior.

Upregulated miR-577 inhibits the Wnt/ $\beta$-catenin signaling pathway by regulating WNT2B in NSCLC cells. To address the miR-577 whether regulates the Wnt/ $\beta$-catenin signaling pathway in NSCLC cells, the expression levels of WNT-associated genes and the activity of $\beta$-catenin were analyzed in the context of miR-577 overexpression or miR-577 overexpression in conjunction with pWNT2B in NSCLC cells. The nuclear distribution of $\beta$-catenin in A549 cells was decreased in pri-miR-577-transfected cells and increased in pWNT2B-transfected cells (Fig. 4A). Western 
Table I. Clinical and pathologic characteristics of patients with non-small cell lung cancer.

microRNA-577

Variables

Total no. (n)

Low (n)

$\operatorname{High}(\mathrm{n})$

$\chi^{2}$

P-value

Age (years)

$\geq 60$

$$
6
$$

8

6

$<60$

8

0.337

Gender

Female

$$
7
$$

6

Tumor size $(\mathrm{cm})$

$\begin{array}{ll}\geq 5 & 11 \\ <5 & 14\end{array}$

9

5

TNM stage

I+II 10

III+IV

$$
3
$$

16

12

2

Negative

Histological type

Squamous

Adenocarinoma
14

11
B

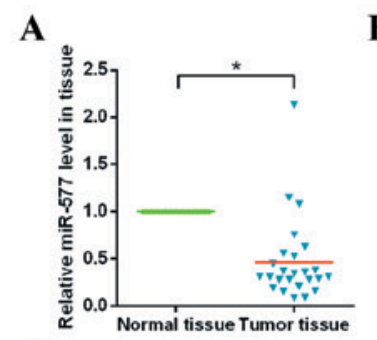

E
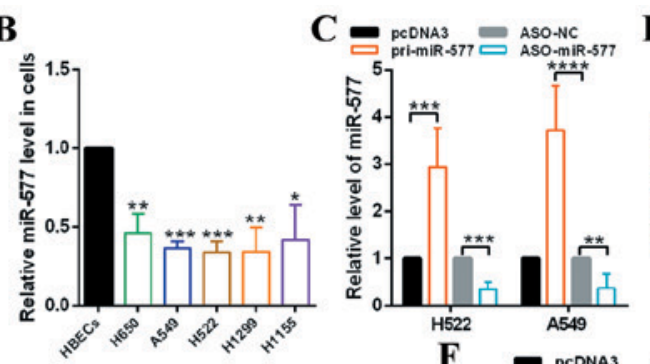

D

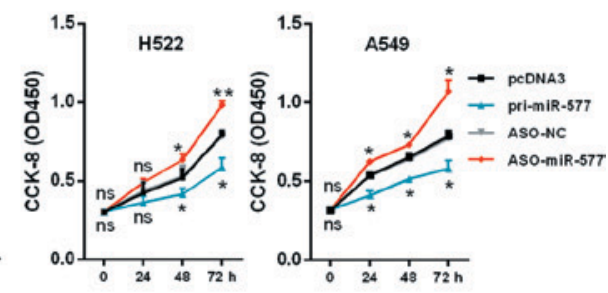

F $={ }^{\text {pCDNA3 }}$ ASO-NC

8

2.231

$8 \quad 3$

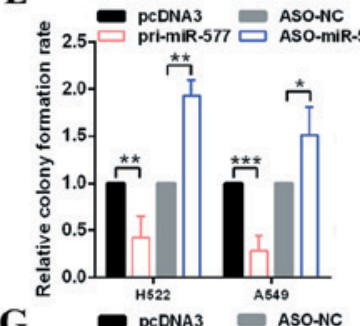

G
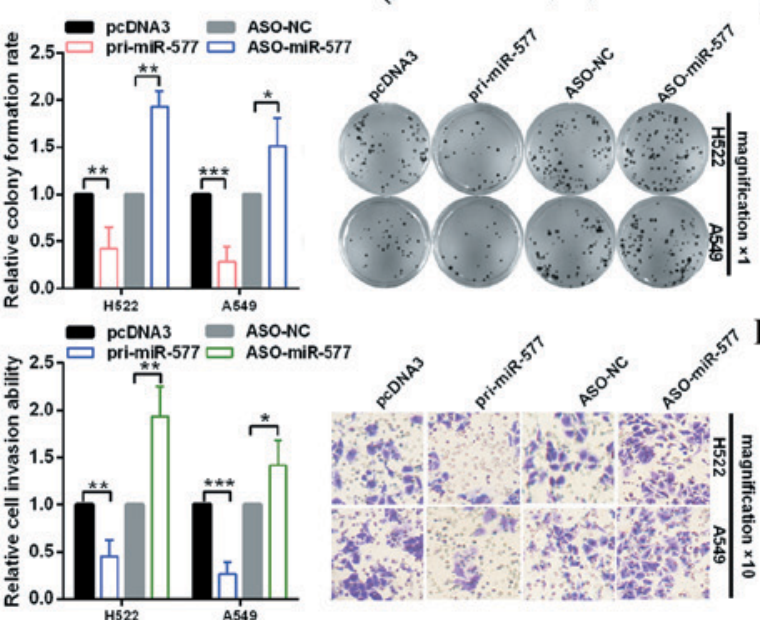

2.5
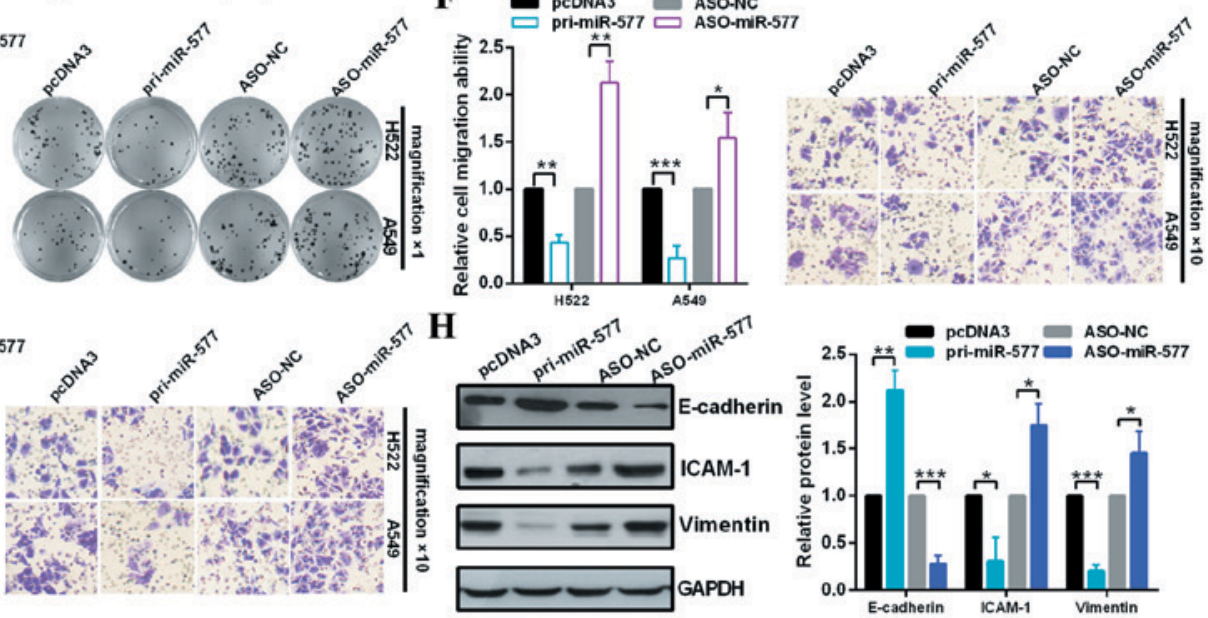

Figure 1. miR-577 functions as a suppressor gene in NSCLC cells. (A) Expression levels of miR-577 in NSCLC and normal tissues were examined by RT-qPCR. (B) Expression levels of miR-577 in HBECs, H650, A549, H522, H1299 and H1155 cells were examined by RT-qPCR. (C) Efficiency of pri-miR-577 or ASO-miR-577 was identified by RT-qPCR. (D) Effect of miR-577 on H522 and A549 cellular viabilities were determined by CCK-8 assay. (E) Relative colony formation rates of $\mathrm{H} 522$ and A549 cells with indicated treatment were determined by colony formation assay. Original magnification, x1. (F) Transwell migration assays revealed that miR-577 suppressed cell migration ability. Original magnification, x10. (G) Transwell invasion assays demonstrated that miR-577 suppressed cell invasion ability. Original magnification, x10. (H) Western blot analysis of the protein expression levels of E-cadherin, ICAM-1 and Vimentin following transfection with pri-miR-577 or ASO-miR-577 and the control groups in A549 cells. *P<0.05; ** P<0.01 vs. control. NSCLC, non-small cell lung cancer; RT-qPCR, reverse transcription-quantitative polymerase chain reaction; miR, microRNA; HBECs, human normal bronchial epithelial cells; ASO, antisense; NC, negative control; CCK-8, cell counting kit-8; ns, no significance; ICAM-1, intercellular adhesion molecule 1. 

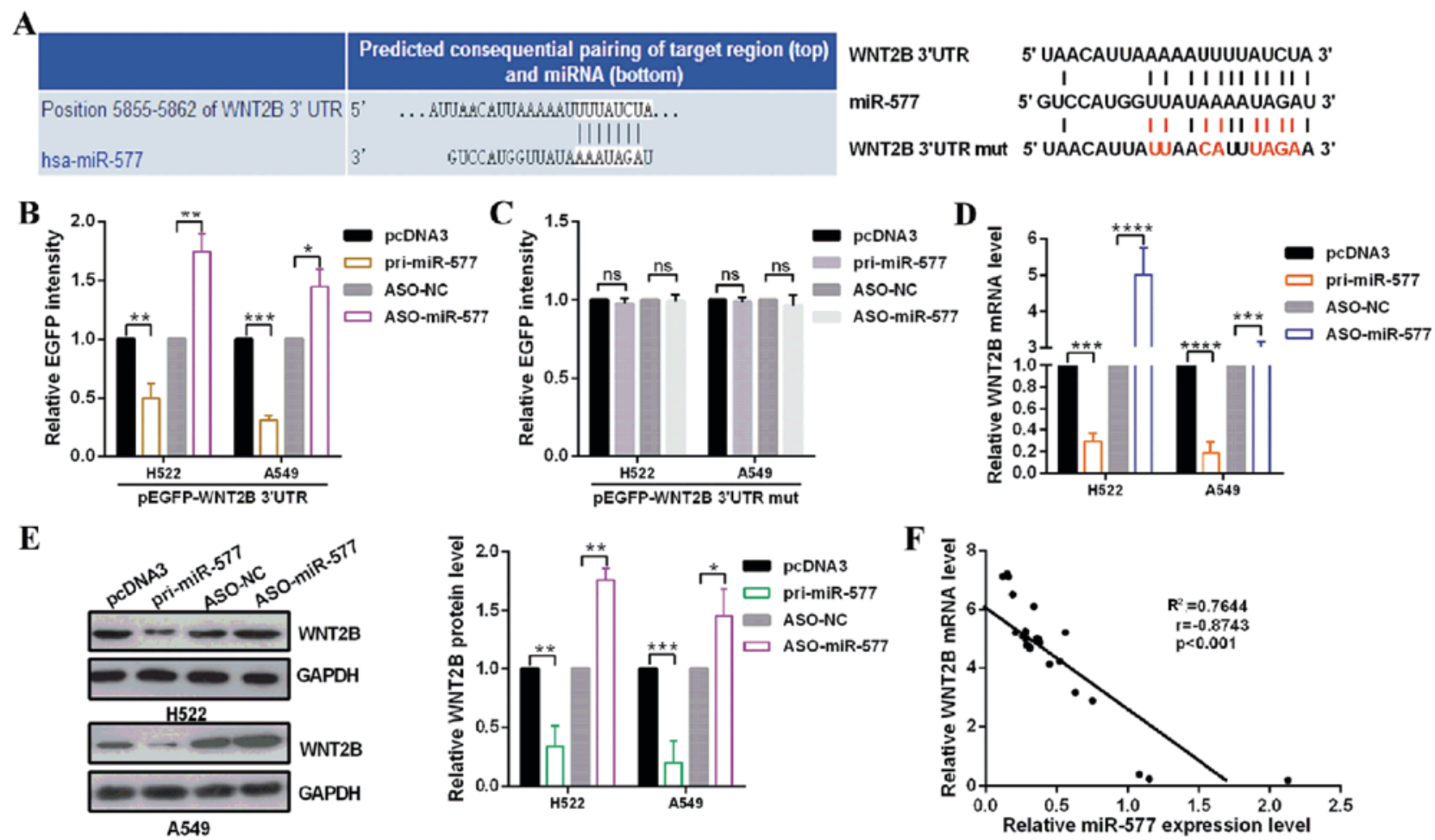

Figure 2. WNT2B is a direct target of miR-577. (A) Predicted miR-577 binding sites in WNT2B mRNA using TargetScan were demonstrated and the mutated 3'UTR of WNT2B mRNA is shown. EGFP intensity of H522 and A549 cells expression the (B) wild type or (C) mutated 3'UTR was determined by spectrophotometry, and the values of control group were set to 1. (D) WNT2B mRNA expression levels in H522 and A549 cells with indicated treatment were measured by RT-qPCR. (E) WNT2B protein level in H522 and A549 cells transfected with pri-miR-577 or ASO-miR-577 and respective controls were determined by western blot analysis. (F) mRNA expression levels of WNT2B was negatively correlated to the miR-577 level by RT-qPCR. ${ }^{*} \mathrm{P}<0.05 ;{ }^{* *} \mathrm{P}<0.01$. WNT2B, Wnt family member 2B; UTR, untranslated region; miR/miRNA, microRNA; mut, mutated; EGFP, enhanced green fluorescent protein; ASO, antisense; $\mathrm{NC}$, negative control; ns, not significant.

blot assays revealed that the expression levels of phosphorylated (p)-GSK3 $\beta$, activated $\beta$-catenin, c-Myc and cyclin D1 were significantly reduced by miR-577 overexpression and significantly increased by WNT2B overexpression (Fig. 4B). In addition, a TOP/FOP flash assay was performed, which is often used to detect $\mathrm{Wnt} / \beta$-catenin signaling. The results demonstrated that miR-577 overexpression decreased the TOP/FOP flash ratio, but WNT2B overexpression increased it in NSCLC cells, indicating inhibition and activation of the Wnt/ $\beta$-catenin signaling pathway, respectively (Fig. 4C).

\section{Discussion}

Dysregulation of miRNAs is important in the genesis and development of various human diseases and cancer, such as NSCLC $(19,20)$. Previous studies have reported that miR-577 is downregulated in numerous cancer types. For example, Jiang et al (21) reported that miR-577 was downregulated in colorectal cancer (CRC) specimens and cell lines and miR-577 overexpression suppressed the proliferation and colony formation and induced a G0/G1 cell cycle arrest in CRC cells. In addition, Chen et al (22) indicated that miR-577 inhibited pancreatic $\beta$-cell function and survival by targeting fibroblast growth factor 21 via the activation of ERK1/2 and AKT signaling pathways in pediatric diabetes. Furthermore, Wang et al (23) reported that miR-577 mimics may repress cell proliferation, enhance cell apoptosis and block the cell cycle in G0/G1 phase in hepatocellular carcinoma. However,
Yuan et al (24) reported that miR-577 overexpression and testis specific 10 downregulation promoted cell proliferation and a more rapid G1-S phase transition in esophageal squamous cell carcinomas. These studies suggested that miR-577 serves important roles in numerous types of human cancer and may be considered as a therapeutic target. Thus, it is important to investigate the role of miR-577 in a variety of cancers. In the present study, the results revealed significant downregulation of miR-577 in NSCLC tissues and cell lines. miR-577 overexpression significantly inhibited cell proliferation, migration and invasion of NSCLC cells in vitro and reduced the expression levels of ICAM-1 and Vimentin and enhancing those of E-cadherin. This suggested that this miRNA may also function as a suppressor gene in NSCLC cells. In addition, miR-577 overexpression inactivated the $\mathrm{Wnt} / \beta$-catenin pathway in NSCLC cells.

It has been reported that miRNAs have roles in a number of cancers via complementary base pairing with the $3^{\prime}$ UTR of their target genes (25). In the present study, bioinformatics were performed to predict target genes; WNT2B may be a novel candidate target of miR-577. Notably, the mRNA expression levels of WNT2B were significantly increased in H650, A549, H522, H1299 and H1155 cell lines, and were inversely correlated with miR-577 expression levels in NSCLC cells. Furthermore, the EGFP reporter assay indicated that miR-577 binds directly to the 3'UTR of WNT2B mRNA within H522 and A549 cells. The results from RT-qPCR and western blot analyses in the present study 


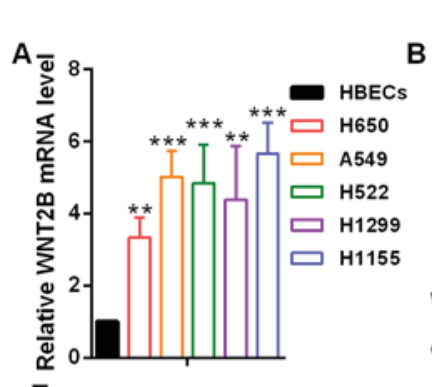

E$$
\text { (1.07 }
$$

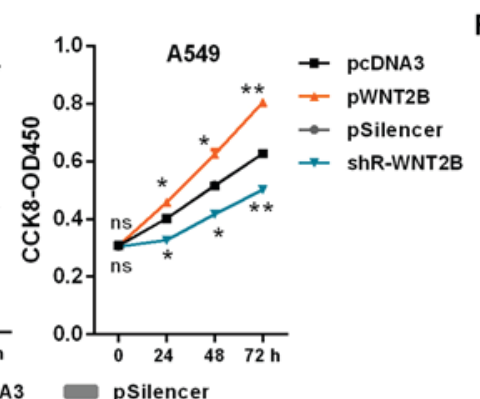

$\mathbf{F}$

G
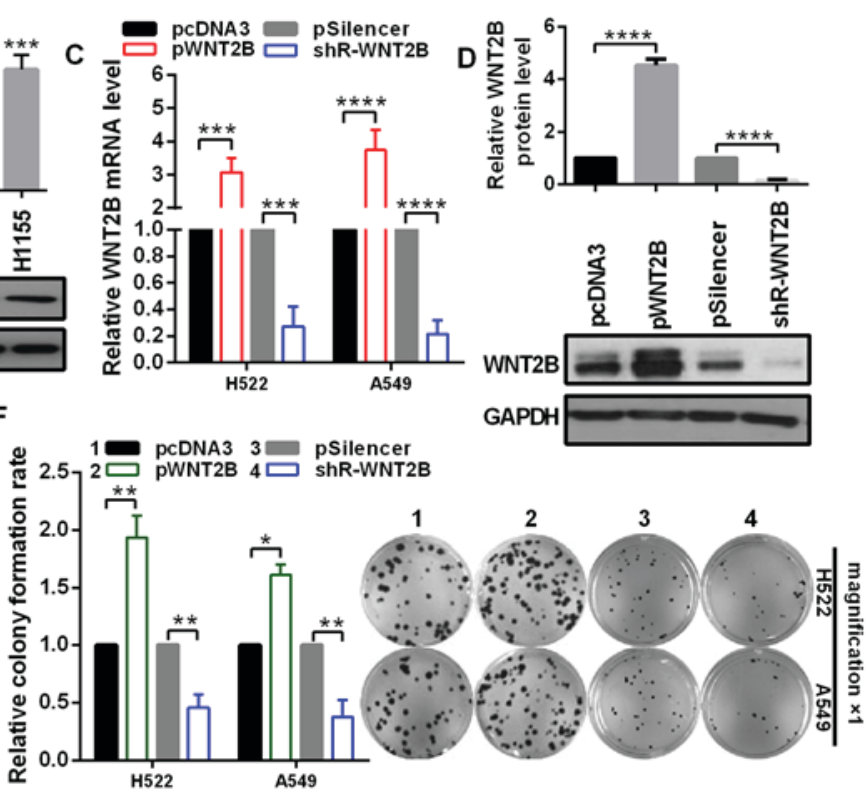
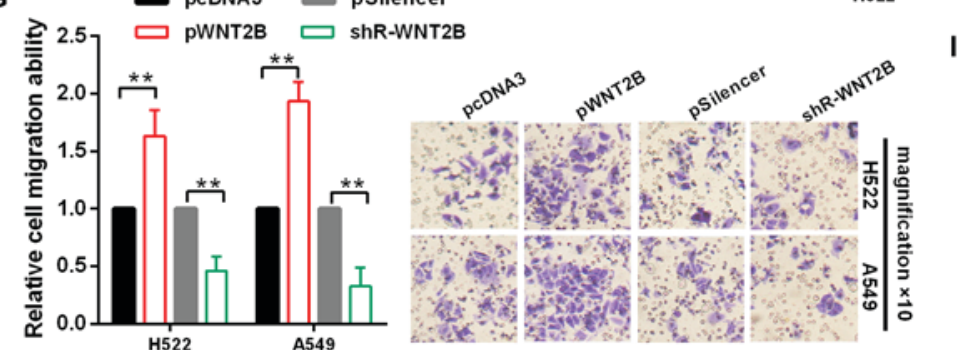

H

- pCDNA3 pSilencer

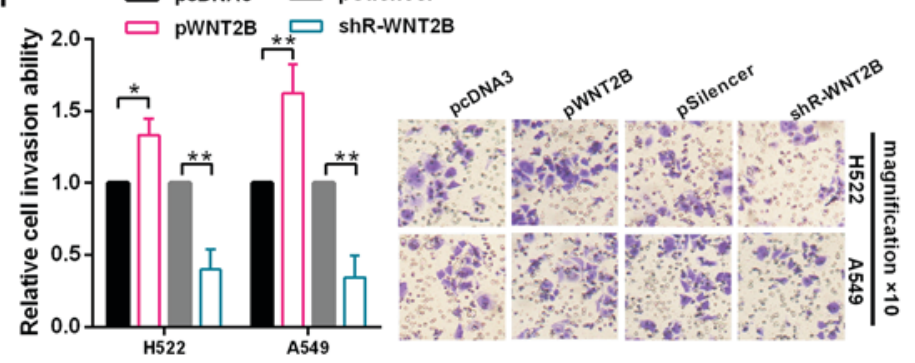

I

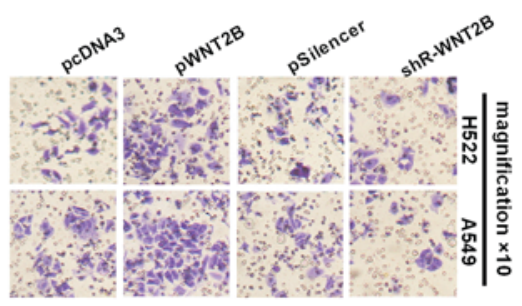

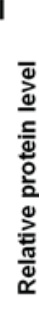
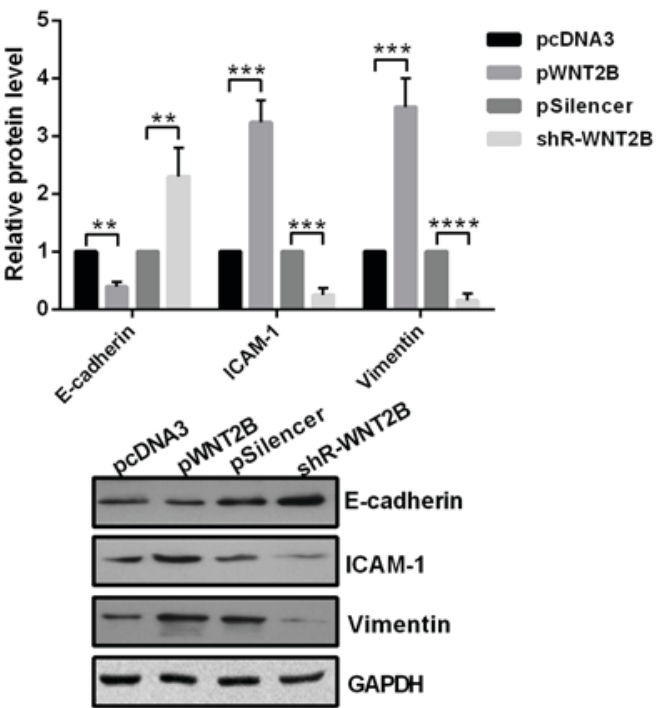

Figure 3. WNT2B promotes the malignant phenotype of non-small cell lung cancer cells. (A) mRNA expression levels of WNT2B in HBECs, H650, A549, H522, H1299 and H1155 cells were examined by RT-qPCR. (B) Protein expression levels of WNT2B in HBECs, H650, A549, H522, H1299 and H1155 cells were examined by western blot analysis. (C) RT-qPCR demonstrated that overexpression and knockdown of WNT2B was efficient. (D) Western blot analysis revealed that overexpression and knockdown of WNT2B was efficient in A549 cells. (E) CCK-8 assay demonstrated that WNT2B overexpression increased cell viability and WNT2B knockdown decreased cell viability. (F) Relative colony formation rate was higher following transfection with pWNT2B and lower with shR-WNT2B compared with the control groups. Original magnification, x1. (G) Transwell migration assays revealed that WNT2B overexpression promoted cell migration ability. Original magnification, x10. (H) Transwell invasion assays revealed that WNT2B overexpression promoted cell invasion ability. Original magnification, x10. (I) Western blot analysis demonstrated the protein expression levels of E-cadherin, ICAM-1 and Vimentin, following transfection with pWNT2B or shR-WNT2B in A549 cells. $\mathrm{P}<0.05 ;{ }^{* *} \mathrm{P}<0.01 ;{ }^{* * *} \mathrm{P}<0.001$. RT-qPCR, reverse transcription-quantitative polymerase chain reaction; WNT2B, Wnt family member 2B; shR, short hairpin RNA; CCK-8, cell counting kit-8; ns, no significance; ICAM-1, intercellular adhesion molecule 1.

revealed that miR-577 overexpression significantly reduced the expression of WNT2B at mRNA and protein levels; miR-577 knockdown significantly increased the expression of WNT2B at mRNA and protein levels in H522 and A549 cells.

The canonical Wnt $/ \beta$-catenin signaling pathway is one of the most critical developmental pathways that are considered important for progenitor cell fate, developmental decisions, proliferation during embryonic development, adult tissue homeostasis and tumor microenvironment (26-28). WNT2B has been reported to stimulate the canonical Wnt $/ \beta$-catenin signaling pathway and affect various malignant tumor progressions (29). In nasopharyngeal carcinoma, WNT2B was significantly upregulated and higher expression of WNT2B was closely correlated with TNM stage, clinic stage and cervical lymph node metastasis (30). Wang et al (31) suggested that WNT2B knockdown inhibited cell colony formation and metastasis and enhanced chemotherapy sensitivity via the caspase-9/Bcl-2/Bcl-xl and EMT/p-AKT signaling pathways in ovarian cancer (31). These studies highlighted the role of WNT2B in malignant processes of tumors; however, the function of WNT2B in NSCLC remains unclear. The results of the present study demonstrated that the mRNA and protein expression levels of WNT2B in 


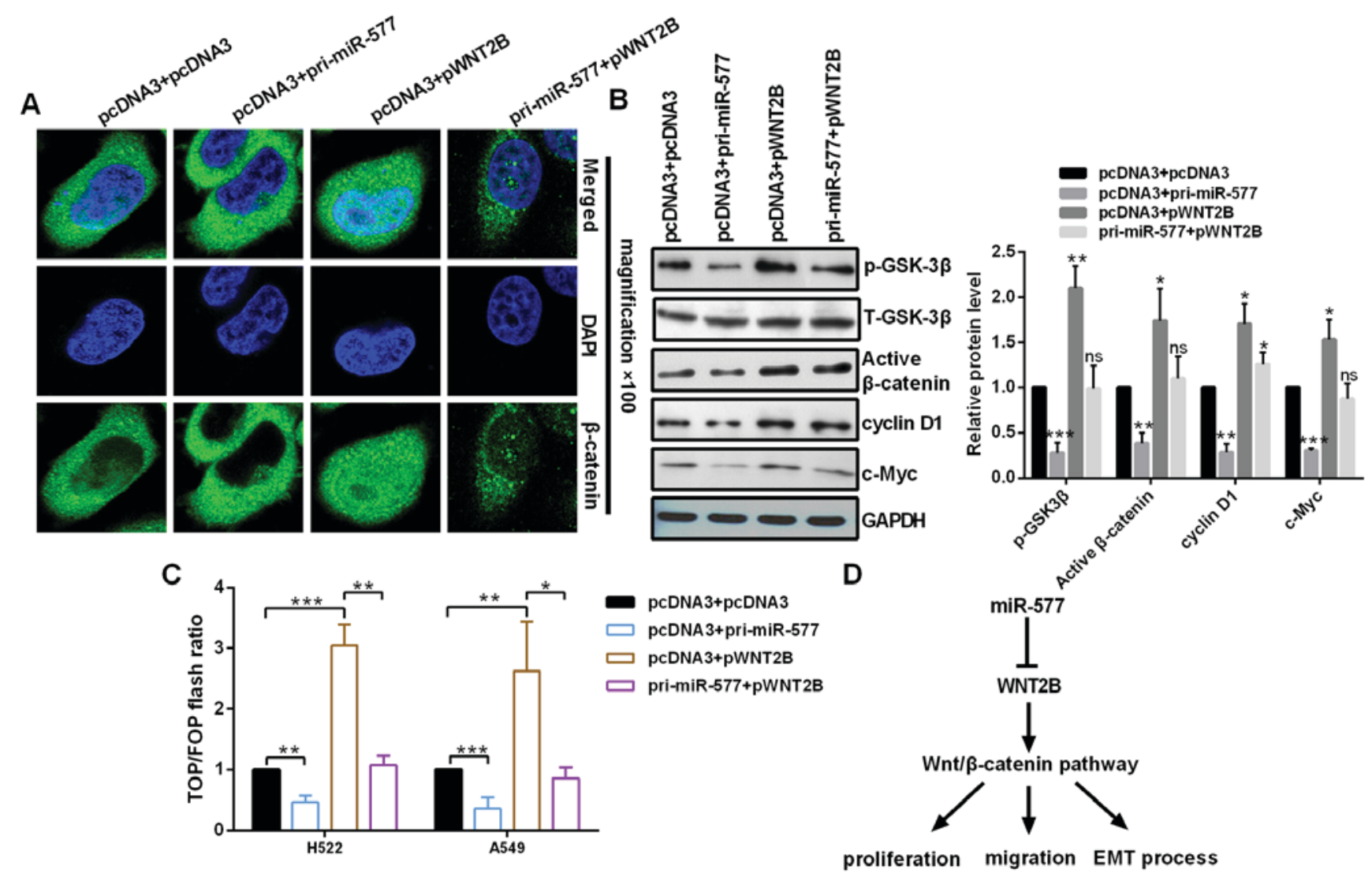

Figure 4. miR-577 negatively regulates the Wnt/ $\beta$-catenin pathway. (A) Immunofluorescence staining was used to examine the nuclear distribution of $\beta$-catenin in A549 cells. Original magnification, $x 100$. (B) Western blot analysis was conducted to detect the expression levels of p-GSK3 $\beta$, total-GSK3 $\beta, \beta$-catenin, c-Myc and cyclin D1 in A549 cells. (C) TOP/FOP luciferase reporter assays were performed to detect the $\beta$-catenin activity in H522 and A549 cells. (D) Regulation model of miR-577. "P $<0.05$; *" $\mathrm{P}<0.01$. miR, microRNA; WNT2B, Wnt family member $2 \mathrm{~B}$; p-GSK3 $\beta$, phospho-glycogen synthase kinase $\beta$; ns, not significant; EMT, epithelial-mesenchymal transition.

NSCLC cell lines were upregulated, and that overexpression of WNT2B may have promoted cell viability, colony formation ability and the EMT process in NSCLC cells. In addition, ectopic WNT2B may activate the Wnt/ $\beta$-catenin pathway in NSCLC cells.

In conclusion, the present study confirmed that the downregulation of miR-577 is a common phenomenon in NSCLC tissues and cell lines, and identified that miR-577 serves an important role in regulating cell proliferation, migration and invasion, and the EMT process in NSCLC cells. In addition, miR-577 may have inhibited WNT2B expression at both the mRNA and protein expression levels by directly targeting the 3'UTR of the WNT2B mRNA. Furthermore, miR-577 may inhibit the activation of the Wnt/ $\beta$-catenin signaling pathway by regulating WNT2B in NSCLC cells (Fig. 4D). Collectively, these findings may provide a novel insight into tumorigenesis and miR-577 may be considered as a potential biomarker for NSCLC.

\section{Acknowledgements}

Not applicable.

\section{Funding}

No funding was received.

\section{Availability of data and materials}

The datasets used and analyzed during the current study are available from the corresponding author on reasonable request.

\section{Authors' contributions}

RJ conceived and supervised the project. BW and RJ performed the experiments. LS and JL analyzed the data. BW and RJ wrote the manuscript. All the authors reviewed the manuscript.

\section{Ethics approval and consent to participate}

The present study was approved by the ethical review committees of Tianjin Huanhu Hospital. All of the samples were obtained with the patients' informed consent.

\section{Patient consent for publication}

All patients provided consent for publication.

\section{Competing interests}

The authors declare that they have no competing interests. 


\section{References}

1. Ferlay J, Shin HR, Bray F, Forman D, Mathers C and Parkin DM: Estimates of worldwide burden of cancer in 2008: GLOBOCAN 2008. Int J Cancer 127: 2893-2917, 2010.

2. Osugi J, Muto S, Matsumura Y, Higuchi M, Suzuki H and Gotoh M: Prognostic impact of the high-sensitivity modified Glasgow prognostic score in patients with resectable non-small cell lung cancer. J Cancer Res Ther 12: 945-951, 2016.

3. Siegel R, Ma J, Zou Z and Jemal A: Cancer statistics, 2014. CA Cancer J Clin 64: 9-29, 2014.

4. Buettner R, Wolf $\mathrm{J}$ and Thomas RK: Lessons learned from lung cancer genomics: The emerging concept of individualized diagnostics and treatment. J Clin Oncol 31: 1858-1865, 2013.

5. Ramnath N, Dilling TJ, Harris LJ, Kim AW, Michaud GC, Balekian AA, Diekemper R, Detterbeck FC and Arenberg DA: Treatment of stage III non-small cell lung cancer: Diagnosis and management of lung cancer, 3rd ed: American college of chest physicians evidence-based clinical practice guidelines. Chest 143 (5 Suppl): e314S-e340S, 2013.

6. Novaes FT, Cataneo DC, Ruiz Junior RL, Defaveri J, Michelin OC and Cataneo AJ: Lung cancer: Histology, staging, treatment and survival. J Bras Pneumol 34: 595-600, 2008.

7. Zaporozhchenko IA, Morozkin ES, Skvortsova TE, Ponomaryova AA, Rykova EY, Cherdyntseva NV, Polovnikov ES, Pashkovskaya OA, Pokushalov EA, Vlassov VV and Laktionov PP: Plasma miR-19b and miR-183 as potential biomarkers of lung cancer. PLoS One 11: e0165261, 2016.

8. Bartel DP: MicroRNAs: Genomics, biogenesis, mechanism, and function. Cell 116: 281-297, 2004.

9. Ameres SL and Zamore PD: Diversifying microRNA sequence and function. Nat Rev Mol Cell Biol 14: 475-488, 2013.

10. Chen CZ: MicroRNAs as oncogenes and tumor suppressors. N Engl J Med 353: 1768-1771, 2005.

11. Yu N, Zhang Q, Liu Q, Yang J and Zhang S: A meta-analysis: MicroRNAs' prognostic function in patients with nonsmall cell lung cancer. Cancer Med 6: 2098-2105, 2017.

12. Chen Y, Lu L, Feng B, Han S, Cui S, Chu X, Chen L and Wang R: Non-coding RNAs as emerging regulators of epithelial to mesenchymal transition in non-small cell lung cancer. Oncotarget 8: 36787-36799, 2017.

13. Legras A, Pécuchet N, Imbeaud S, Pallier K, Didelot A, Roussel H, Gibault L, Fabre E, Le Pimpec-Barthes F, LaurentPuig $\mathrm{P}$ and Blons H: Epithelial-to-mesenchymal transition and MicroRNAs in lung cancer. Cancers (Basel) 9: pii: E101, 2017.

14. Sun Y, Li L, Xing S, Pan Y, Shi Y, Zhang L and Shen Q: miR503-3p induces apoptosis of lung cancer cells by regulating p21 and CDK4 expression. Cancer Biomark 20: 597-608, 2017.

15. Li Y, Zhang H, Dong Y, Fan Y, Li Y, Zhao C, Wang C, Liu J, Li X, Dong M, et al: MiR-146b-5p functions as a suppressor miRNA and prognosis predictor in non-small cell lung cancer. J Cancer 8: 1704-1716, 2017.
16. Huang LX, Hu CY, Jing L, Wang MC, Xu M, Wang J, Wang Y, Nan KJ and Wang SH: microRNA-219-5p inhibits epithelial-mesenchymal transition and metastasis of colorectal cancer by targeting lymphoid enhancer-binding factor 1. Cancer Sci 108: 1985-1995, 2017.

17. Livak KJ and Schmittgen TD: Analysis of relative gene expression data using real-time quantitative PCR and the 2(-Delta Delta C(T)) method. Methods 25: 402-408, 2001.

18. Wong N and Wang X: miRDB: An online resource for microRNA target prediction and functional annotations. Nucleic Acids Res 43 (Database Issue): D146-D152, 2015.

19. Hou J, Meng F, Chan LW, Cho WC and Wong SC: Circulating plasma MicroRNAs as diagnostic markers for NSCLC. Front Genet 7: 193. eCollection, 2016.

20. Del Vescovo V and Denti MA: microRNA and lung cancer. Adv Exp Med Biol 889: 153-177, 2015.

21. Jiang H, Ju H, Zhang L, Lu H and Jie K: microRNA-577 suppresses tumor growth and enhances chemosensitivity in colorectal cancer. J Biochem Mol Toxicol 31: 2017.

22. Chen XY, Li GM, Dong Q and Peng H: MiR-577 inhibits pancreatic $\beta$-cell function and survival by targeting fibroblast growth factor 21 (FGF-21) in pediatric diabetes. Genet Mol Res 14: 15462-15470, 2015.

23. Wang LY, Li B, Jiang HH, Zhuang LW and Liu Y: Inhibition effect of miR-577 on hepatocellular carcinoma cell growth via targeting $\beta$-catenin. Asian Pac J Trop Med 8: 923-929, 2015.

24. Yuan X, He J, Sun F and Gu J: Effects and interactions of MiR-577 and TSGA10 in regulating esophageal squamous cell carcinoma. Int J Clin Exp Pathol 6: 2651-2667, 2013.

25. Farazi TA, Juranek SA and Tuschl T: The growing catalog of small RNAs and their association with distinct Argonaute/Piwi family members. Development 135: 1201-1214, 2008.

26. Majidinia M, Aghazadeh J, Jahanban-Esfahlani R and Yousefi B: The roles of $\mathrm{Wnt} / \beta$-catenin pathway in tissue development and regenerative medicine. J Cell Physiol 233: 5598-5612, 2018.

27. Murillo-Garzón V and Kypta R: WNT signalling in prostate cancer. Nat Rev Urol 14: 683-696, 2017.

28. Nusse R and Clevers $\mathrm{H}$ : Wnt/ $/$-catenin signaling, disease, and emerging therapeutic modalities. Cell 169: 985-999, 2017.

29. Katoh M: Differential regulation of WNT2 and WNT2B expression in human cancer. Int J Mol Med 8: 657-660, 2001.

30. Liu C, Li G, Yang N, Su Z, Zhang S, Deng T, Ren S, Lu S, Tian Y, Liu Y and Qiu Y: miR-324-3p suppresses migration and invasion by targeting WNT2B in nasopharyngeal carcinoma. Cancer Cell Int 17: 2, 2017.

31. Wang H,Fan L, Xia X, Rao Y,MaQ, Yang J,Lu Y, Wang C, Ma D and Huang X: Silencing Wnt2B by siRNA interference inhibits metastasis and enhances chemotherapy sensitivity in ovarian cancer. Int J Gynecol Cancer 22: 755-761, 2012.

This work is licensed under a Creative Commons Attribution-NonCommercial-NoDerivatives 4.0 International (CC BY-NC-ND 4.0) License. 